\title{
Study on Tail Characteristics of Systemic Risk in US and Chinese Stock Markets
}

\author{
Yujie Lai ${ }^{1,2, a}$, Yibo $\mathrm{Hu}^{1, \mathrm{~b}}$ \\ 1. School of Economic management, Xi'an Aeronautical University, Xi'an 710077, China \\ 2. School of Automation, Northwestern Polytechnical University, Xi'an 710072, China \\ a laiyujie811014@163.com, b huyiboo@163.com
}

\begin{abstract}
Trade friction between the United States and China has increased financial risks. Since 2017, the stock markets of the United States and China have shown extremely high-risk dependence. By constructing Markov SJC Copula model, this paper makes an empirical analysis on the systemic risk of American and Chinese stock markets. The results show that SJC Copula can describe the systemic risk of American and Chinese stock markets well. In addition, studies show that the risk dependence of the U.S. and Chinese stock markets also has obvious tail dynamic characteristics. Under the high-risk zone system, the dependence of the lower tail risk is more significant.
\end{abstract}

Keywords: Systematic Risk; Tail Correlation; Markov Mechanism Transformation.

\section{Introduction}

Previous studies have mainly examined linear and symmetric systemic risks under normal market conditions. However, under extreme market conditions, it is very important to investigate systemic risks and their possible tail nonlinearity, asymmetry and dynamic characteristics [1]. In view of this, this article uses the Copula method to capture and measure systemic risk in the US and Chinese stock markets. The Copula method is an important method in the study of the correlation structure of financial market variables. It can flexibly and effectively capture the complex correlation structure between financial market variables. Such as nonlinear correlation, tail correlation under extreme market conditions, asymmetric correlation and dynamic correlation, etc. [2]. In addition, Copula allows modeling the marginal distribution and correlation structure of financial market variables respectively, and constructs complex non-normal joint distribution by selecting different marginal distribution and Copula. This increases the flexibility of modeling and helps to fully characterize the non-normal characteristics of financial market variables such as "skew", "leptokurtosis and fat-tail". Therefore, Copula has attracted many scholars' attention in the application of finance [3].

In this paper, by examining the tail correlation structure of stock market volatility in the United States and China under extreme market conditions, selecting the appropriate Copula function and constructing the corresponding Markov mechanism to transform the Copula model, the systemic risk in the United States and China stock market is studied, and the possible asymmetric and tail dynamic characteristics of risk dependence are investigated.

\section{Model Parameter Estimation}

In this paper, IFM (Influence Function for Margins) method is used to estimate Markov mechanism transformation Copula model. In the first stage, the marginal distribution of stock return volatility is estimated by nonparametric method; in the second stage, the marginal distribution is substituted into copula function to estimate copula parameter. According to existing research, the two-stage method is a very effective estimation method and is easy to handle in calculation [4]. In addition, semiparametric estimation can avoid the marginal distribution from specification error and obtain stable Copula parameter estimation results [5].

In order to fully describe the empirical characteristics of the volatility of stock returns, such as "skew", "leptokurtosis and fat-tail", this paper estimates the marginal distribution function $F\left(x_{t}\right)$ by 
nonparametric method. Specifically, the marginal distribution function is estimated based on the rescaled empirical cumulative distribution function:

$$
\hat{F}\left(x_{t}\right)=\frac{1}{T+1} \sum_{t=1}^{T} 1\left\{x_{t}<x\right\}
$$

Where $1\{\cdot\}$ is an illustrative function. The rescaling of the empirical cumulative distribution function is to ensure that the first-order conditions of Copula log-likelihood function define all finite T's. According to Glivenko-Cantelli theorem, $\hat{F}(x)$ converges uniformly to the real empirical cumulative distribution function $F(x)$.

In Markov mechanism switching Copula model, parameter vector $\theta=\left(p, q, \theta_{0}^{\prime}, \theta_{1}^{\prime}\right)$ to be estimated. Given the nonparametric estimation of the empirical cumulative distribution function of volatility, the maximum likelihood method is used to obtain the estimation of Markov mechanism transformation Copula model parameters as follows:

$$
\hat{\theta}=\arg \max _{\theta} \hat{\zeta}
$$

In the formula, $\hat{\zeta}=\sum \ln c^{M R S}\left(\hat{F}\left(x_{t}\right), \hat{F}\left(x_{t-1}\right) \mid \theta\right)$ is a log-likelihood function, calculated iteratively according to the following system:

$$
\begin{gathered}
\hat{\xi}_{t \mid t}=\frac{\hat{\xi}_{t \mid t-1} \odot \eta_{t}}{\left(\hat{\xi}_{t \mid t-1}\right)^{\prime} \eta_{t}} \\
\hat{\xi}_{t+1 \mid t}=P^{\prime} \hat{\xi}_{t \mid t} \\
\eta_{t}=\left\{\begin{array}{l}
c\left(\hat{F}\left(x_{t}\right), \hat{F}\left(x_{t-1} \mid \theta_{0}\right)\right. \\
c\left(\hat{F}\left(x_{t}\right), \hat{F}\left(x_{t-1} \mid \theta_{1}\right)\right.
\end{array}\right. \\
c^{M R S}\left(\hat{F}\left(x_{t}\right), \hat{F}\left(x_{t-1} \mid \theta\right)\right)=\left(\hat{\xi}_{t \mid t-1}\right)^{\prime} \eta_{t}
\end{gathered}
$$

Where, $\odot$ is Hadamard product; the initial value $\hat{\xi}_{0 \mid 0}$ is set as unconditional probability (ergodic probability), that is:

$$
\hat{\xi}_{0 \mid 0}=\left\{\begin{array}{l}
(1-q) /(2-p-q) \\
(1-p) /(2-p-q)
\end{array}\right.
$$

\section{Empirical Research}

\subsection{Data and Descriptive Statistics}

In this paper, the volatility data of the daily return rate of the STANDARD \& Poor's Index (SP500) and shanghai-Shenzhen 300 index (HS300) are used as research samples. The time span of data is From January 4, 2002 to November 29, 2019. By eliminating the data with inconsistent trading days to make trading days match, the sample data is finally determined to be 4206 groups. All data are from Wind database. First, take logarithmic difference processing for data, that is, $r_{t}=\ln P_{t}-\ln P_{t-1}, \mathrm{P}$ is closing price. The volatility of return rate adopts the method of return sequence variance. 


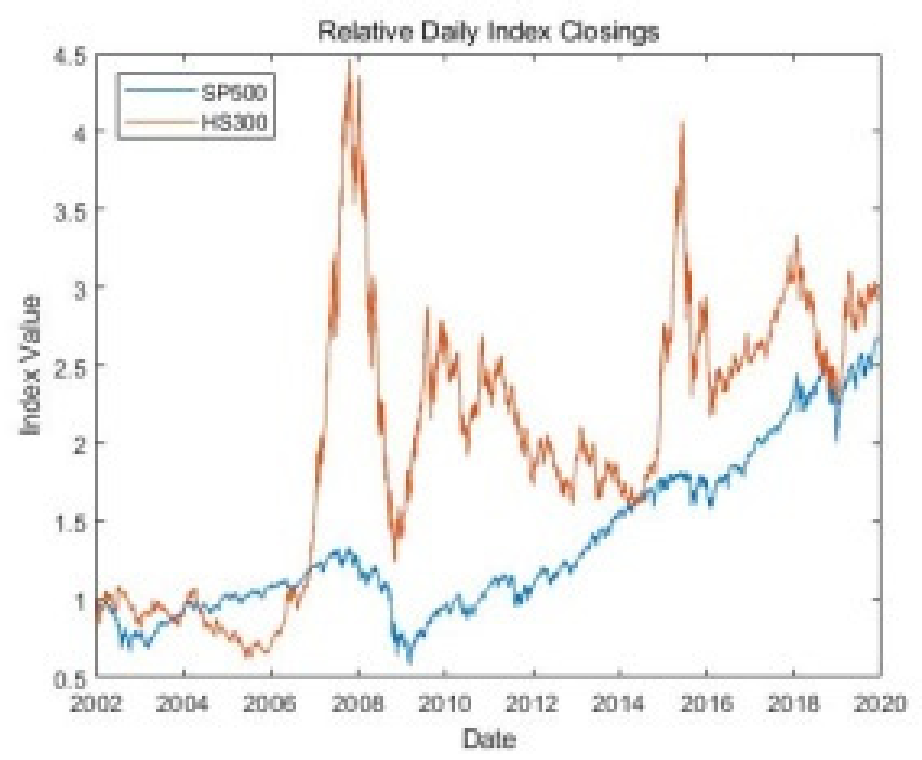

Fig 1. Normalized daily volatility of SP500 and HS300

Figure 1 shows the relative volatility of the two-stock market standardized indexes in the past 20 years. Assets all show the phenomenon of increased volatility, with the Shanghai and Shenzhen 300 indexes experiencing the most severe volatility. It shows that the two selected assets show the characteristics of mean regression, and alternate into low volatility and high volatility.

\subsection{Markov SJC Copula Parameter Estimation Results}

In order to obtain the possible tail dynamic characteristics of risk dependence in the U.S. and China's stock markets, SJC copula with the best fitting effect is selected, and the corresponding Markov mechanism transformation copula model is constructed for analysis. Using IFM estimation method, the parameter estimation results of the model are obtained, as shown in Table 1. Using Hamilton algorithm, the lower and upper tail dynamic processes of stock market volatility in the United States and China are calculated.

$$
\lambda^{\mathrm{k}}(t)=\operatorname{Pr}\left(s_{t}=0 \mid I_{t-1}\right) \lambda_{0}^{k}+\operatorname{Pr}\left(s_{t}=1 \mid I_{t-1}\right) \lambda_{1}^{k}, \quad k=L, U
$$

Table 1. Parameter estimation results of SJC Copula model transformed by Markov mechanism

\begin{tabular}{|c|c|c|c|c|}
\hline & Transfer probability & Duration probability & $\lambda^{L}$ & $\lambda^{U}$ \\
\hline Regime 0 & 0.8209 & 0.1791 & 0.1112 & 0.0635 \\
\hline Regime 1 & 0.1499 & 0.8501 & 0.0011 & 0.0010 \\
\hline
\end{tabular}

It can be seen from table 1 that SJC copula model shows better fitting ability after introducing mechanism transformation. By comparing the duration probability and the transfer probability, it is found that regime $0\left(s_{t}=0\right)$ is a high-risk dependent region, and its duration and transfer probability are 0.8209 and 0.1791 , respectively. Regime $1 \quad\left(s_{t}=1\right)$ is a low-risk dependent region with a probability of persistence and transition of 0.1499 and 0.8501 , respectively. Under $s_{t}=0$, the risk dependence of U.S. and China's stock market is 0.1112 at the bottom and 0.0635 at the top. Under $s_{t}=1$, the bottom tail correlation of risk dependence of us and China stock market is 0.0010 , and the top tail correlation is 0.0011 . Whether $s_{t}=0$ or $s_{t}=1$, the bottom tail correlation degree of American and Chinese stock markets is greater than the top tail correlation degree. At the same time, it is found 
that the correlation coefficients of the lower tail and the upper tail of the U.S. and Chinese stock markets in regime 0 are much larger than those of regimel.

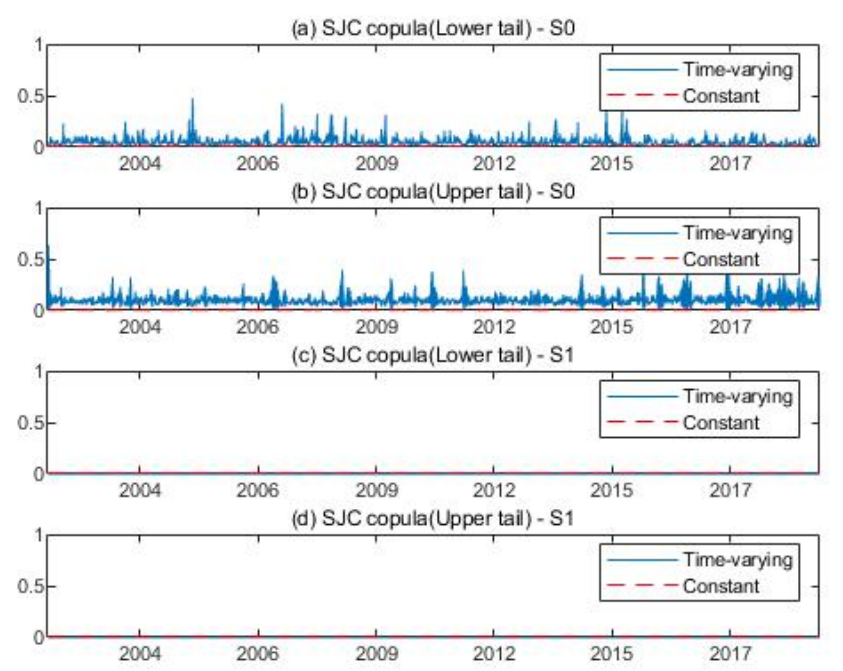

Fig 2. Dynamic correlations between the bottom and top tails of SP500 and HS300

It can be seen from fig. 2 that when $s_{t}=1$, the upper tail risk and the lower tail risk in the United States and China are not significant. When $s_{t}=0$, the top-end risk and bottom-end risk of Chinese and American stock markets are both at a higher level, indicating that tail risks are more likely to occur when risks are highly dependent. Among them, the period with higher upper tail risk appears in 2005 and 2015, and the period with higher lower tail risk appears in 2006, 2016 and 2017 to date. This is also in line with the actual situation. Figure 2 shows that the U.S. and Chinese stock markets are in a high tail risk since 2017, which is also the main reason for the high degree of risk dependence between the two.

\section{Conclusion}

The persistence probability of US and Chinese stock markets in regime 0 is much smaller than that in regime1. This shows that most of the time, the US and Chinese stock markets are in a low-risk dependent regime. The financial crisis that broke out in 2008 intensified the linkage between global stock markets. After the financial crisis broke out, the tail correlation between the U.S. and China stock market indexes has been significantly enhanced, which also confirms that the global financial storm triggered by the U.S. subprime mortgage crisis has caused a structural change in the correlation of the stock market. Since 2017, the trade friction between the United States and China has increased the financial risk. Since 2017, the stock market of the United States and China has shown a high-risk dependence. In the high-risk dependent region, the upper tail and lower tail risks show time-varying characteristics. The period with higher upper tail risk appears in 2005, 2015, and the period with higher lower tail risk appears in 2006, 2016, and 2017 to date. This shows that the U.S. and Chinese stock markets are in a high tail risk, which is also the main reason for the high degree of risk dependence between the two.

\section{Acknowledgments}

Thanks to the research project of General project of University-level scientific Research Fund of $\mathrm{Xi}$ 'an Aeronautical University (2020KY2233); Higher Education Research Project of Xi 'an Aeronautical University (2021GJ1009); Higher Education Scientific research project of Shaanxi Province(XGH21232). 


\section{References}

[1] Ning C, Xu D, Wirjanto T S. Is volatility clustering of asset returns asymmetric?, Journal of Banking \& Finance. Vol. 52 (2015), 62-76.

[2] Rodriguez J C. Measuring financial contagion: A copula approach, Journal of Empirical Finance. Vol. 14 (2007), 401-423.

[3] Jondeau E, Rockinger M. The copula-garch model of conditional dependencies: An international stock market application, Journal of International Money and Finance. Vol. 25 (2006), 827-853.

[4] Patton A J, Rockinger M: Copula-based Models for Financial Time Series (Springer, 2009), p. 767-785.

[5] Bielecki T R, Cousin A, Crépey S, et al. Dynamic hedging of portfolio credit risk in a Markov copula model, Journal of Optimization Theory and Applications. Vol. 161 (2014), 90-102. 